Swarthmore College

Works

\title{
The Effect Of Speaker-Specific Information On Pragmatic Inferences
}

Daniel J. Grodner

Swarthmore College, dgrodne1@swarthmore.edu

J. Sedivy

Follow this and additional works at: https://works.swarthmore.edu/fac-psychology

Part of the Psychology Commons

Let us know how access to these works benefits you

\section{Recommended Citation}

Daniel J. Grodner and J. Sedivy. (2011). "The Effect Of Speaker-Specific Information On Pragmatic Inferences". Processing And Acquisition Of Reference. 239-271.

https://works.swarthmore.edu/fac-psychology/531

This work is brought to you for free by Swarthmore College Libraries' Works. It has been accepted for inclusion in Psychology Faculty Works by an authorized administrator of Works. For more information, please contact myworks@swarthmore.edu. 
The Processing and Acquisition of Reference

edited by Edward A. Gibson and

Neal J. Pearlmutter

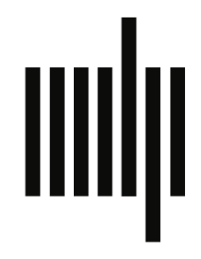

The MIT Press
From The MIT Press

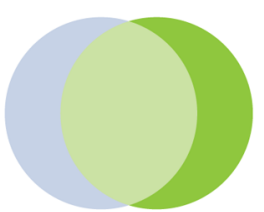

MITCogNet 
(C) 2011 Massachusetts Institute of Technology

All rights reserved. No part of this book may be reproduced in any form by any electronic or mechanical means (including photocopying, recording, or information storage and retrieval) without permission in writing from the publisher.

For information about quantity discounts, email special_sales@mitpress.mit.edu.

Set in Times New Roman and Syntax on InDesign by Asco Typesetters, Hong Kong. Printed and bound in the United States of America.

Library of Congress Cataloging-in-Publication Data

The processing and acquisition of reference / edited by Edward A. Gibson and Neal J. Pearlmutter.

p. $\mathrm{cm}$.

This volume presents papers from the special session at the CUNY Sentence Processing conference that was hosted by MIT and Northeastern University in 2003. Includes bibliographical references and index.

ISBN 978-0-262-01512-7 (alk. paper)

1. Reference (Linguistics) - Congresses. 2. Language acquisition-Congresses. I. Gibson, Edward, 1962- II. Pearlmutter, Neal J., 1967-

P325.5.R44P76 2011

$401^{\prime} .456-\mathrm{dc} 22$

$\begin{array}{llllllllll}10 & 9 & 8 & 7 & 6 & 5 & 4 & 3 & 2 & 1\end{array}$ 


\title{
10 The Effect of Speaker-Specific Information on Pragmatic Inferences
}

\author{
Daniel Grodner and Julie C. Sedivy
}

It is commonplace to observe that utterances can convey more information than they explicitly encode. Indeed, the field of pragmatics has grown from the insight that speakers exploit communicative conventions in order to say more with less. Less attention has been paid to the burden this places on perceivers. Because speakers' meanings are underspecified, perceivers must infer implicit content in order to interpret, and successfully situate, utterances within the current discourse. These inferences ${ }^{1}$ appeal to many types of knowledge, including entailment relations, world knowledge, and discourse context. The last of these presents a particular challenge to investigators because contexts are dynamic and utterance meaning can be sensitive to context. For instance, the utterance in (2) implies something like (2a) if it is a response to (1a) and something like (2b) if it is a response to (1b). ${ }^{2}$

(1) a. What time is it?

b. How good is the party?

(2) Some guests are already leaving.

a. It must be late.

b. The party is not much fun.

The ease and the prevalence of such inferences facilitate efficient communication but create difficulty for models of language understanding. A central puzzle is how the extrasentential context is combined with intrasentential information to ultimately yield an interpretation of a sentence.

The present paper addresses this issue by exploring a particular dependency between the referential environment and linguistic form. Suppose a speaker wishes to refer to one member of a set of entities belonging to the same nominal category in the current discourse. The speaker must use a modified expression in order to refer successfully. For instance, if one cup is the intended referent in a context containing two cups, the speaker must use a restrictive 
modifier, such as "the cup on the left" or "the red cup." Significantly, this dependence appears to be bidirectional. When a listener encounters a restrictively modified referential description, such as the tall cup, two sets are invoked in the immediate discourse: a target set corresponding to the literal denotation of the expression (e.g., a tall cup) and a contrast set containing an entity of the same type as the noun but differing along the dimension picked out by the adjective (e.g., a short cup).

Indirect evidence for the contrastive inference comes from studies of structural ambiguity resolution. In general, the sentence-processing mechanism prefers syntactic alternatives that contain simple unmodified descriptions when given the option. Crain and Steedman (1985) proposed that this preference stems from the fact that modified structures occasion costly changes to the discourse model. As an illustration, consider the string The horse raced past the barn ..., which is ambiguous between a main clause containing a simple NP subject and a reduced relative clause (RC) modifying the subject. The simple NP reading presupposes the existence of a single referent set, namely a horse, in the current discourse model. The complex NP reading involves the projection of an additional contrasting set of entities that share the properties denoted by the head noun but differ by virtue of the property expressed in the modifier. For the present example this corresponds to a non-empty set of horses that were not raced past the barn. In a null context, the modified NP reading requires the greatest number of additions to the current discourse model in order to be felicitous. Thus, the simple NP is preferred. There is evidence that by establishing appropriate referent and contrast sets it is possible to alter parsing preferences (Altmann, Garnham, and Dennis 1992; Altmann and Steedman 1988; Sedivy 2002; Spivey-Knowlton and Tanenhaus 1994).

More direct evidence of contrastive inference comes from studies that monitor perceivers' eye movements as they rearrange objects according to spoken instructions (Tanenhaus, Spivey-Knowlton, Eberhard, and Sedivy 1995; Sedivy, Tanenhaus, Chambers, and Carlson 1999; Sedivy 2003). Sedivy et al. had individuals respond to instructions containing a prenominally modified phrase, such as "Pick up the tall cup." Object arrays consisted of four entities: a target object (e.g., a tall cup), a competitor from a different nominal category that shared the modifier property of the target (e.g., a tall pitcher), an irrelevant distracter object, and either a contrasting object of the same category as the target but possessing a distinct modifier property (e.g., a short cup) or a second distracter. Figure 10.1 depicts a sample display. Note that an indeterminacy is introduced when the modifier is uttered. The input to this point is compatible with reference to either the target or the competitor. If perceivers are driven by the literal meaning of the modifier, their attention could be directed to either 


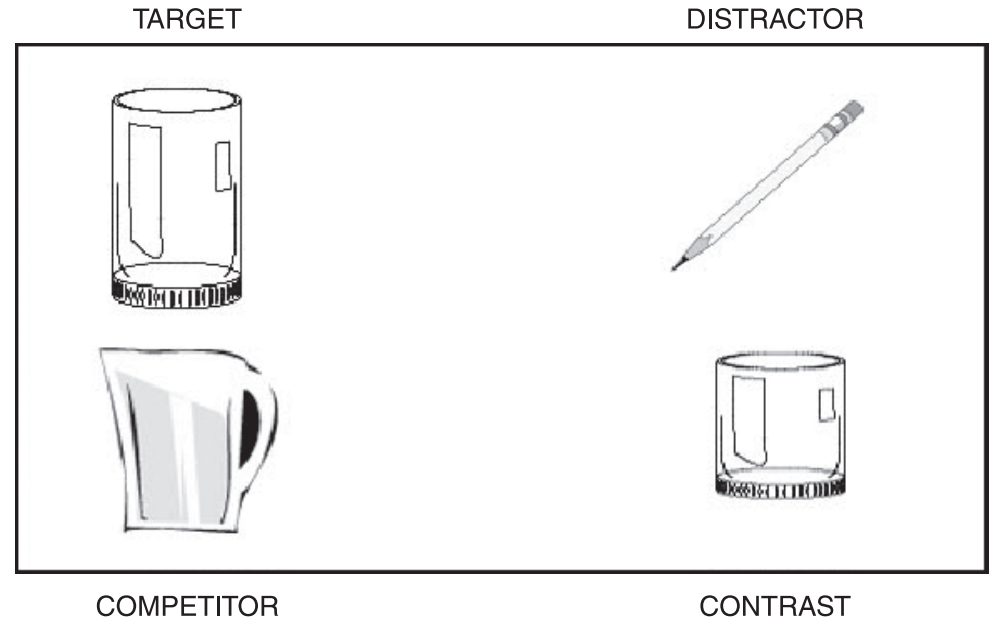

Figure 10.1

Sample contrast condition display for the target instruction "Pick up the tall glass." Each display contained four objects. In the "no contrast" display the contrast was replaced by a second distracter object.

referent. However, if they interpret the modifier contrastively, they should attend to the target object, as this object requires a modified description in order to distinguish it from the contrasting object of the same category. Consistent with this, individuals made earlier fixations on the target and fewer fixations on the competitor in the presence of a contextual contrast. The influence of referential contrast on eye movements has been observed extremely early in the speech stream - within $200 \mathrm{msec}$ of the onset of the head noun (Sedivy 2003). In light of estimates that saccadic eye movements are planned 150-200 msec before being launched (Matin, Shao, and Boff 1993), restrictive modifiers can generate the expectation for referential contrast well before the nominal head can be identified. Because the speech input does not uniquely isolate the target before utterance of the noun, perceivers' fixations must be guided by inferential content.

Two types of accounts have been forwarded to explain the effect of discourse contrast: form-based and pragmatic. Form-based accounts attribute the contrastive inference to the conventional content of restrictively modified descriptions. This type of explanation has the advantage that it requires consideration of information contained only within the sentence. Perceivers would not have to deliberate over various types of extrasentential information. This comports well with the speed and the automaticity of contrast effects.

One way for the conventional meaning to engender a contrastive inference is if definite modified NP structures conventionally presuppose contrasting 
entities in much the same way that a definite description might be said to conventionally presuppose uniqueness of reference. This idea was suggested by Steedman and Altmann (1989). However, this account is difficult to maintain in light of examples like (3), which indicate that the contrastive inference is cancelable in a way that conventionally expressed content is not (Grice 1975; Sadock 1978).

(3) Use the tall cup because there are no other cups.

Perhaps a more plausible way to play out a form-based presuppositional account is to propose that modificational content is by default placed in focus. Focused material is typically analyzed as foregrounded against a background of contrasting alternatives (Rooth 1985, 1992). Thus, for instance, focusing the modifier for the string above as in The horse RACED PAST THE BARN ... would make prominent a single horse that was raced past the barn against a presupposed background of a set of horses that were not raced past the barn. Note that a focus-mediated presuppositional account is consistent with the occurrence of modifiers in the absence of referential contrast. This is because modifiers need not always be placed in focus, and unfocused material is not predicted to generate a set of alternatives.

Reading-time results from studies examining syntactic ambiguities lend some support to the focus-mediated interpretation of referential effects. In particular, the presence of the overt focus operator "only" can induce a preference for ambiguous material to be analyzed as a nominal modifier ( $\mathrm{Ni}$, Crain, and Shankweiler 1996; Sedivy 2002; but see Clifton, Bock, and Radó 2000). However, it is difficult to extend this explanation to spoken stimuli involving prenominal modification (e.g. "Pick up the tall cup."). For these utterances, focus marking would result in greatest prominence on the adjective. However, referential contrast effects were found with spoken stimuli in which greatest prominence occurred on the head noun, according to rules of nuclear stress placement. Hence, the referential-contrast effect does not seem to depend on focus marking.

Another form-based possibility is that the effect of referential contrast is lexically driven. For instance, scalar adjectives, such as tall, are inherently relational and must be evaluated relative to a contextually salient comparison class. Clearly what counts as tall for a child is very different from what counts as tall for an elephant (Siegel 1980; Bierwisch 1987). It might be a lexical idiosyncrasy of scalar modifiers that they cause perceivers to attend inordinately to the discourse context and, thereby, receive contrastive interpretations. This provides an account for the referential-contrast effect with scalar adjectives, but does not extend to referential effects with other modifiers, such as the 
postnominal modification involved in syntactic ambiguity resolution, or even referential effects found for non-scalar modifiers, such as modifiers denoting material properties (Sedivy 2001). Thus, at the very least, any form-based approach seems to require a combination of at least two somewhat distinct mechanisms to account for the range of effects observed to date.

Perhaps the most striking evidence against any of the above accounts, either singly or in combination, is evidence that whether a modifier invokes a contrast can depend on what it modifies. Sedivy (2003) examined the processing of descriptions that contained prenominal color modifiers in the presence or absence of a contrasting object. In one condition, the adjective denoted a property that was highly predictable from the noun category, as in "the yellow banana." In another, the color property was not strongly associated with the noun, as in "the yellow cup." The presence of a contextual contrast aided individuals in identifying objects whose colors were predictable. For instance, individuals more quickly identified a yellow banana, and directed fewer eye movements to other yellow objects in the display, in the presence of a green banana. However, there was no benefit of contextual contrast conferred when identifying objects whose color properties were unpredictable. Individuals' eye movements did not converge more quickly on a yellow cup in the presence of a green cup. The contrastive inference is therefore not an inherent part of the meaning of the color modifier but rather is related to its informativity with respect to the entity being modified.

Such informativity effects are predicted to arise within a pragmatic explanation of referential contrast (Clifton and Ferreira 1989; Sedivy 2003). The pragmatic account claims that contrastive inferences arise because the use of a restrictive modifier is embedded in a collaborative communicative exchange. There is an understanding between discourse participants that speakers are only as informative as they need to be. This follows from Grice's second maxim of quantity $(1975$, p. 26): "Don't make your contribution more informative than is required for the purposes of the present exchange." A simple NP would suffice to pick out the intended referent in a context with only one entity. The use of a more prolix or unusual form indicates that a different state of affairs prevails (see Levinson 2000 for an extended treatment of this idea). The most natural way to make the inclusion of the modifier informative is to ascribe a distinguishing function to it. To apply this logic, perceivers must reason counterfactually about what the speaker could have said but chose not to say.

In support of the pragmatic view, contrastive inferences arise only for adjective types that are not used to label objects in isolation. Scalar modifiers and predictable color modifiers are rarely encoded in default descriptions, whereas unpredictable color modifiers are frequently encoded in default descriptions 
(Sedivy 2000, 2002). The connection between inferential patterns in comprehension and distributions in production suggests that perceivers actively generate expectations as to what referential form a speaker will opt for in selecting an entity. Deviations from those expectations result in pragmatic inferences.

The appeal of this Gricean mechanism is that it is capable of adapting appropriately to a wide range of stimulus conditions. It also provides a unified explanation for all the referential-context effects found to date. On the other hand, it requires a set of as yet unspecified computations that appeal to heterogeneous and potentially unconstrained knowledge bases. Many different factors might be weighed in deciding whether a modified form should be interpreted contrastively. These include the intrinsic properties of a referent, the discourse context, the reliability of the speaker, the intentions of the speaker, the common background of the interlocutors, the goals of the communicative situation, and expectations about alternative forms. It is unlikely that perceivers could consider all of these in the limited time frame in which contextual contrast effects have been observed. In fact, Clifton and Ferreira argued that such conversational implicatures would be too cumbersome for individuals to engage in on-line, and that, hence, an expeditious parser would not consider such information in initial interpretive processes.

The studies described above establish only that listeners are sensitive to the use of default descriptions when inferring a contrast. This need not imply that individuals are engaged in full-blown Gricean reasoning. It may be that certain inferential steps are short-circuited. This resembles the position of the socalled neo-Griceans (Gazdar 1979; Horn 1984; Levinson 2000). These researchers propose a principled division between conversational implicatures that are generalized across situations and those that are specific to certain situations. For instance, the implicatures in (2a) and (2b) are dependent on the particular context in which (2) is uttered. However, the scalar implicature in (2c) is present across of the vast majority of discourses.

(2) c. Not all of the guests are leaving.

Levinson (2000) hypothesizes that generalized implicatures like this one form part of the meaning for certain utterance types, whereas particularized implicatures are associated with utterance tokens. For quantity-based implicatures, the form type selected by a speaker is located on an informativity scale with respect to some anticipated reference type (Horn 1971, 1989). This comparison process can generate an implicature when the selected form is less informative than an alternative. For instance, the selection of "some" in (2) triggers a comparison with the more informative scalar alternative "all." The failure to use the more constraining alternative results in the implicature (2c). Analogously, 
contrastive inferences arise when the form selected is more informative than an anticipated alternative.

The generality and simplicity of this comparison mechanism suggest that quantity-based inferences could arise through automatic processes rather than being computed on the fly. If the routines for projecting a contrast are precompiled in this way, this could explain the immediacy with which the visual context influences reference resolution. It also predicts that generalized inferences might be harder to block or suspend than particularized ones such as (2a) and (2b). Motivated by computational constraints, Jurafsky (2003) outlines a similar idea to explain how perceivers interpret what speech act a speaker intended to perform. In this model, perceivers use surface cues probabilistically to realize the illocutionary force of an utterance. Because the implicit content arises from statistical associations, cues that are highly reliable indicators of implicit content should be harder to ignore. In the present example, this reasoning might predict an insensitivity to the situation in which the modifier is used. Because default descriptions are statically linked with individual referents, contrastive inferences do not require that perceivers consider the particular circumstances of the immediate discourse. Perceivers might reflexively infer a contrast whenever the speaker deviates from a stored default form.

An alternative is proposed by Carston (1998). Building on the work of Sperber and Wilson (1986), he claims that all conversational inferences are actively constructed and therefore particularized to their circumstances. Such nonce inference is clearly a necessary component of language comprehension in order to explain the robustness of the inferences made in (2a) and (2b). What is at issue is whether this nonce mechanism is involved in the earliest stages of interpretive processing.

The experiment described below makes an effort to distinguish the shortcircuited and nonce inference positions by manipulating characteristics idiosyncratic to a particular speaker - specifically, the degree to which the speaker could be considered to be adhering to normative conversational principles. If contrastive inferences are generalized implicatures triggered by deviations from a default form, the effect of contrast should be impervious to this manipulation, at least with respect to initial referential commitments. On the other hand, if contrastive inferences are the product of pragmatic reasoning about particular discourse situations, there should be a noticeable effect when the circumstances are altered in this critical way.

\section{Experiment}

There is a great deal of evidence that speakers make many accommodations that demonstrate their sensitivity to the specific needs of a listener. For example, 
experts adjust referential forms appropriately to the knowledge state of the addressee (Isaacs and Clark 1987), and speakers hyper-articulate to make speech clearer under conditions where intelligibility is likely to be reduced for a particular listener (Bradlow 2002). Knowledge specific to a conversational partner is also a potentially powerful constraint in comprehension. For instance, suppose (4) is uttered discourse initially.

(4) He's such a jerk.

Resolving the antecedent of the pronoun depends critically on discerning what potential referent is most likely to be salient for the individual speaker. In extreme cases, an utterance such as (4) may be effortlessly resolved even when uttered out of the blue as the first interaction in days or even weeks between interlocutors, and with no particular supporting visual context if the interlocutors share knowledge of some male person extraordinarily inclined to behave badly.

Few studies have investigated the moment-to-moment application of speaker-related knowledge in comprehension. The few studies that have investigated this have employed head-mounted eye tracking to monitor visual attention as listeners perform a target-identification task. One important finding that emerges from this work is that listeners are sensitive to the speaker's perspective in the referential discourse. Listeners exhibit preferential consideration for entities in the shared referential environment over items to which they have privileged access (Hanna, Tanenhaus, and Trueswell 2003; Nadig and Sedivy 2002; but see Barr and Keysar 2002 and Barr 2003). Hanna et al. (2003) provide a particularly powerful illustration of this ability. They show that listeners are sensitive to the speaker's discourse model even when it is at odds with the perceptually available referential environment. These findings illustrate that speaker-based information can be recruited very quickly to resolve potential referential indeterminacies. It is interesting to note, however, that these studies exploit the conventional properties of the referring expression (in these cases, the uniqueness requirement of definite descriptions) to signal the need to constrain reference. It has been argued that circumscribing a referential domain is central to communication (Chambers et al. 2004; Hanna et al. 2003; Tanenhaus 2003). The requirement that definite descriptions refer to a uniquely identifiable entity, when combined with an experimental scenario in which satisfying this requirement necessitates accessing speaker-based information, creates optimal conditions for observing powerful and rapid speaker-based effects.

It is interesting to extend such research to the question of whether perceivers access speaker-based information even when the conventional properties of the referring expression can be satisfied without recourse to speaker-based 
information. Metzing and Brennan (2003) reported the use of speaker-based knowledge to interpret referring expressions in just such a situation. They found that perceivers were sensitive to the way a particular speaker had chosen to refer to an object in the past, and that they used this information inferentially. When the same speaker used a different but equally plausible label for an item, looks to that object were delayed, and there were more fixations to other objects in the display before converging on the target referent. Participants expected the new label to refer to a new object and interrogated the scene in an attempt to find it. This may have indicated that a pragmatic inference was arrived at when individuals tried to find a relevant function for the departure from an entrained label (cf. Grice's maxim of manner). No such penalty was observed when a new speaker used the novel label. Participants were equally quick to respond to the new or the old label when the label was uttered by a new speaker. However, one must be cautious before concluding that these findings demonstrate immediate use of speaker identity in inferencing. On average, the first fixations to the target object were launched approximately one second after the onset of the referring expression. This is slower than the latencies seen in comparable tasks, and it raises the possibility that participants adopted specialized strategies or that eye movements were being driven by non-primary processes. Still, it demonstrates that individuals are capable of applying the pragmatic expectation that a speaker will use a specific entrained description at some point in reference resolution.

The present experiment extends the result of Metzing and Brennan by looking at whether perceivers generate expectations about the type of referring expression a speaker will use and then use this as the basis for generating inferences of referential contrast. We constructed a scenario in which a particular speaker does not obey the standard communicative conventions. Specifically, a number of cues were given to indicate that the speaker's use of restrictive modification was not a reliable signal of the presence of a contextual contrast. If the contrastive inference is predicated on the perceiver's belief that the speaker rationally chose one linguistic form over another, the contrast effect should be defeated in this situation. If instead the inference is a reflex of generalized and automatic informativity expectations, this manipulation should not affect the projection of a contrast, and the presence of a contextual contrast should aid listeners in identifying the target.

A further manipulation in the present study was to look at the differential properties of material and scalar modifiers. Material properties are not context dependent in the same way as scalar properties. Nevertheless, just as for scalar adjectives and redundant color adjectives, the referents for descriptions containing material modifiers are easier to identify in the presence of a contextual 
contrast (Sedivy 2001). One possibility is that the different modifiers engender a contrastive inference in disparate ways. Adjectives that denote inherently relational properties, such as scalar terms, might engender a contrast effect as a result of their lexical semantics. Modifiers that have no special relation to the context of their use, such as material and color terms, might instead convey contrast pragmatically. If so, then the expectation for contextual contrast given by scalar terms might be less susceptible to being blocked by the present pragmatic manipulation than the expectation for contextual contrast given by material items.

\section{Participants}

Thirty-one members of the Brown University community were paid for their participation in the experiment. Each was a native speaker of English and had normal or corrected-to-normal vision. All were naive with respect to the goals of the experiment.

\section{Materials and Design}

The methodology and the design were adapted from Sedivy et al. (1999). Participants were asked to manipulate arrays of four objects according to a set of prerecorded instructions. Twenty stimulus sets similar to that illustrated in figure 10.1 were constructed. For each display array there were two to four instructions. A critical instruction containing a prenominally modified noun (e.g., "Pick up the tall cup") always occurred first in the series. In ten of the twenty critical phrases, the prenominal modifier referred to a scalar property. For the other ten, the modifier described a material property (e.g., "the glass mug"). Table 10.1 lists the contrast, competitor, and target objects for the stimuli. For the scalar items, the competitor object was selected so that it was a better exemplar of the modifier property than the target (e.g., an unusually tall pitcher). Thus, if there was an early bias toward the literal interpretation of the adjective, it should have resulted in elevated erroneous looks to the competitor rather than the target. This could not be done for the material items. Half of the experimental items in each session included a contrasting object, and half did not. Each participant saw only the contrast variant or the no-contrast variant of any stimulus set. An additional ten trials contained displays that were like those in the contrast condition except that a modified noun was used to refer to the competitor item. These counterbalancing trials were intended to ensure that individuals would not be cued to expect a modified noun to refer to a member of the contrasting pair. Stimuli and counterbalancing trials were pseudorandomly ordered with 26 filler trials. 
Table 10.1

Experimental items employed in the study.

\begin{tabular}{|c|c|c|c|c|c|}
\hline $\begin{array}{l}\text { Modifier } \\
\text { type }\end{array}$ & Target & Contrast & Competitor & Distracter 1 & Distracter 2 \\
\hline Scalar & Small pad & Large pad & Small doll & Razor & Shampoo \\
\hline Scalar & $\begin{array}{l}\text { Thin } \\
\text { marker }\end{array}$ & Thick marker & Thin brush & $\begin{array}{l}\text { Potato } \\
\text { masher }\end{array}$ & Crayons \\
\hline Scalar & $\begin{array}{l}\text { Thick } \\
\text { notebook }\end{array}$ & $\begin{array}{l}\text { Thin } \\
\text { notebook }\end{array}$ & Thick book & Rag & Horseshoe \\
\hline Scalar & Tall cup & Short cup & Tall pitcher & Eraser & Diskette \\
\hline Scalar & $\begin{array}{l}\text { Narrow } \\
\text { Post-its }\end{array}$ & Wide Post-its & $\begin{array}{l}\text { Narrow } \\
\text { ribbon }\end{array}$ & $\begin{array}{l}\text { Peanut } \\
\text { butter }\end{array}$ & Pink bow \\
\hline Scalar & $\begin{array}{l}\text { Long } \\
\text { envelope }\end{array}$ & $\begin{array}{l}\text { Short } \\
\text { envelope }\end{array}$ & Long spatula & Toy shovel & Lotion \\
\hline Scalar & Tall doll & Short doll & Tall mug & Black pen & $\begin{array}{l}\text { Yellow } \\
\text { folder }\end{array}$ \\
\hline Scalar & Long spoon & Short spoon & Short pencil & Peach & Quarter \\
\hline Scalar & Wide tape & Narrow tape & Wide belt & Banana & Red pencil \\
\hline Scalar & Fat crayon & Thin crayon & Fat marker & Egg & Tupperware \\
\hline Material & Brass frame & Wood frame & $\begin{array}{l}\text { Brass candle } \\
\text { holder }\end{array}$ & Fork & Thread \\
\hline Material & $\begin{array}{l}\text { Leather } \\
\text { glove }\end{array}$ & Wool glove & $\begin{array}{l}\text { Leather } \\
\text { wallet }\end{array}$ & Blue bow & $\begin{array}{l}\text { Tape } \\
\text { measure }\end{array}$ \\
\hline Material & Paper plate & Plastic plate & Paper bag & Salt shaker & Shot glass \\
\hline Material & $\begin{array}{l}\text { Porcelain } \\
\text { bowl }\end{array}$ & Plastic bowl & $\begin{array}{l}\text { Porcelain } \\
\text { saucer }\end{array}$ & Candle & Sunglasses \\
\hline Material & $\begin{array}{l}\text { Plastic } \\
\text { spoon }\end{array}$ & Metal spoon & Plastic comb & Tie & Battery \\
\hline Material & $\begin{array}{l}\text { Styrofoam } \\
\text { ball }\end{array}$ & Rubber ball & $\begin{array}{l}\text { Styrofoam } \\
\text { cup }\end{array}$ & Comb & Lego \\
\hline Material & Metal ladle & Plastic ladle & Metal pan & Orange & Toy octopus \\
\hline Material & Wool sock & Cotton sock & Wool cap & Duct tape & Plastic ruler \\
\hline Material & $\begin{array}{l}\text { Wood } \\
\text { cutting } \\
\text { board }\end{array}$ & $\begin{array}{l}\text { Plastic cutting } \\
\text { board }\end{array}$ & Wood ruler & $\begin{array}{l}\text { Ribbon } \\
\text { spool }\end{array}$ & Mirror \\
\hline Material & Glass mug & Ceramic mug & Glass vase & Pencil & Floss \\
\hline
\end{tabular}


All participants were told that the experimental instructions had been generated by "an individual who was asked to direct a listener through a sequence of object configurations" and that the experiment had been designed to test how effectively individual speakers were able to convey instructions by observing a perceiver's responses. Fifteen participants were assigned to the reliablespeaker condition. The remaining sixteen participants were assigned to the unreliable-speaker condition. The impression of unreliability was conveyed in three ways. First, participants were told that the speaker who had recorded the instructions had an "impairment that caused language and social problems." Second, the speaker described objects and locations erroneously. Five times over the course of the experiment an object was mislabeled. (For instance, a toothbrush was called a "hairbrush.") On three occasions the instructions directed the perceiver to move an object to a location that did not exist. (For instance, a destination might be described as above object $\mathrm{A}$ and below object $\mathrm{B}$, when in fact object B was below object A.) Both of these error types occurred in a minority of the nearly 200 instructions. Third, the speaker consistently used over-informative descriptions. There were 234 nonpronominal references where an unmodified form would have sufficed to indicate the object of interest. Of these, 197 contained a superfluous modifier. The remaining 37 descriptions were unmodified nominal descriptions.

Note that the presence of the modifier was not a reliable cue to the presence of a contextual contrast for the reliable speakers either. Overall, participants in the reliable-speaker condition heard thirty prenominally modified descriptions: the twenty stimuli and ten counterbalancing trials (the latter included with the specific intent of eliminating any within-experiment correlations between modification and contrastive reference). For each participant, only ten of these (the number of stimuli presented in the contrast condition) were uttered in the presence of a contextual contrast for the target. Although the reliable speaker generated far fewer modified forms than the unreliable speaker (30 versus 207), there was no contingency between modification and contextual contrast over the course of the experiment for either speaker.

To avoid placing prominence on the prenominal modifier, nuclear stress was placed on the head noun of the NP in critical instructions. Durations of the adjective and noun are listed in table 10.2. Note that critical regions were comparable across the unreliable-speaker conditions and the reliable-speaker conditions.

\section{Procedure}

Display changes took approximately 5 seconds, and participants were permitted to watch the display as it was being changed. Every display contained a 


\section{Table 10.2}

Duration of modifier and noun in target instructions across conditions, in milliseconds. (Standard errors in parentheses.)

\begin{tabular}{llllll}
\hline & \multicolumn{2}{l}{ Scalar } & & & \multicolumn{2}{l}{ Material } & \\
\cline { 2 - 3 } \cline { 6 - 6 } & Unreliable & Reliable & & Unreliable & Reliable \\
\hline Adjective & $288(18)$ & $289(8)$ & & $351(42)$ & $372(39)$ \\
Noun & $378(37)$ & $373(33)$ & & $332(36)$ & $355(42)$ \\
\hline
\end{tabular}

centrally located fixation cross. Each trial began with a request for the subject to look at the cross, and participants were instructed to rest their eyes on the central cross between instructions. This was done so that eye movements to the target objects could be measured from a default position that was equidistant from all of the objects in the display. Participants were told to follow the instructions as quickly and accurately as they could.

While the participant followed instructions to move objects in the workspace, eye-movement data were recorded using a lightweight ISCAN headmounted video-based tracking system. The camera provided an infrared image of the eye, and determined monocular eye position by monitoring the locations of the center of the pupil and the cornea reflection. A scene camera was mounted on the side of the helmet, providing an image of the subject's field of view. Calibration was carefully monitored throughout each trial, and minor adjustments were occasionally made between trials. A VCR record consisting of the instructions recorded with a microphone and the participant's moment-bymoment gaze fixation superimposed over the scene-camera image, with timecode stamps at $30 \mathrm{~Hz}$, was made for each experimental trial. Because the scene camera was mounted onto the helmet itself, and moved with the participant's head, the VCR record took into account any head movements made by the participant, allowing for unrestricted head and body movements throughout the experiment. The entire experiment, including introduction to the equipment and task, practice and experimental trials, and debriefing, took approximately 35 minutes.

Eye movements were analyzed by playing the audio-video record back for each 33-millisecond frame on a Sony DSR-30 digital VCR. For the experimental trials, critical points in the speech stream were identified, corresponding to the onsets of the adjective and head noun, and the offset of the head noun. Continuous eye movements occurring from the beginning of the instruction were noted until the participant reached for the target object. Thus, the joint identification of critical points in the speech stream and the eye-movement data 


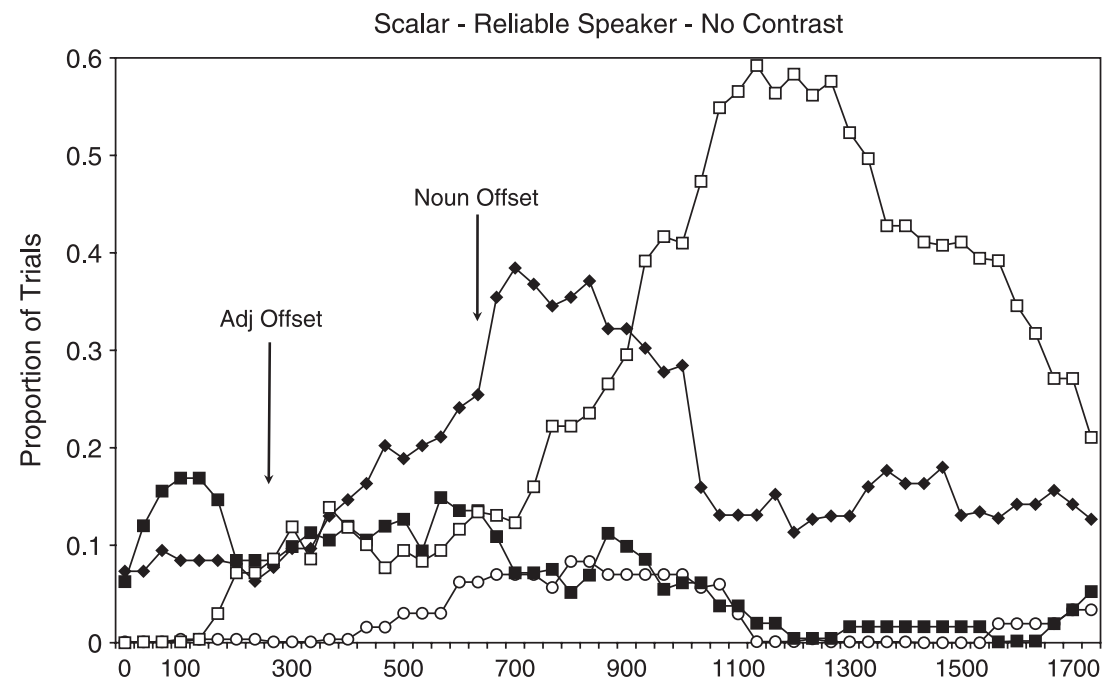

Scalar - Reliable Speaker - Contrast

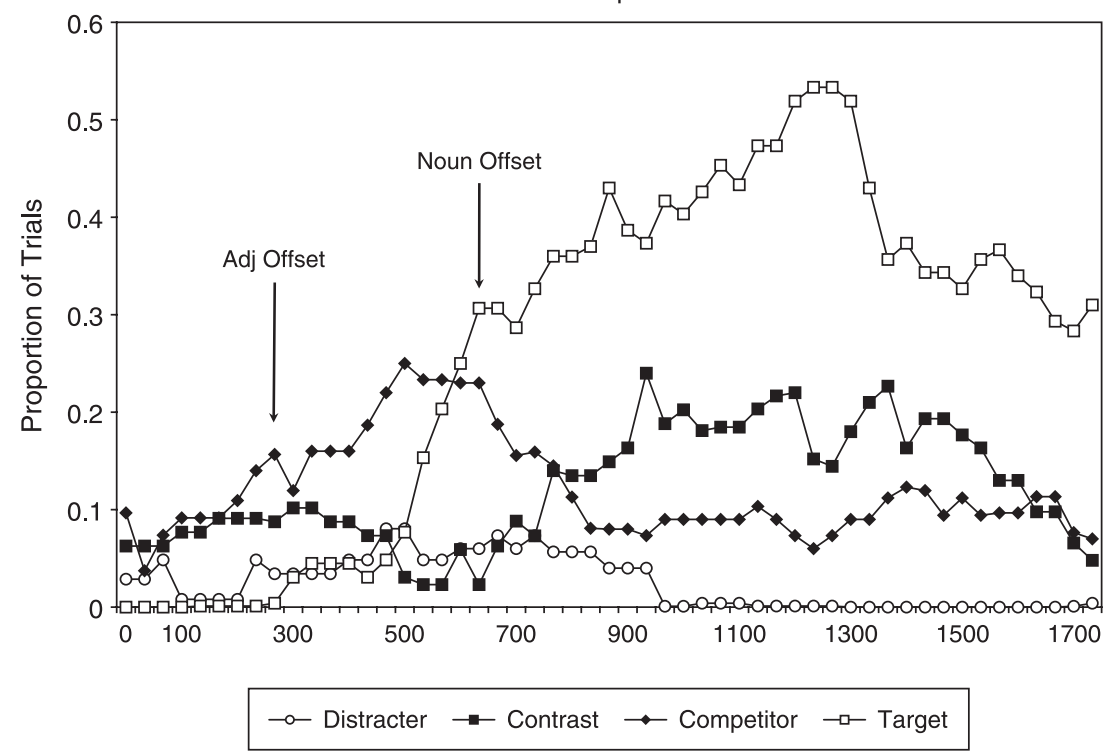

Figure 10.2

Eye-movement record in real time for scalar items. Horizontal axis depicts msec after adjective onset. In the no-contrast conditions the data labeled "contrast" indicate the second distracter item in the display. 

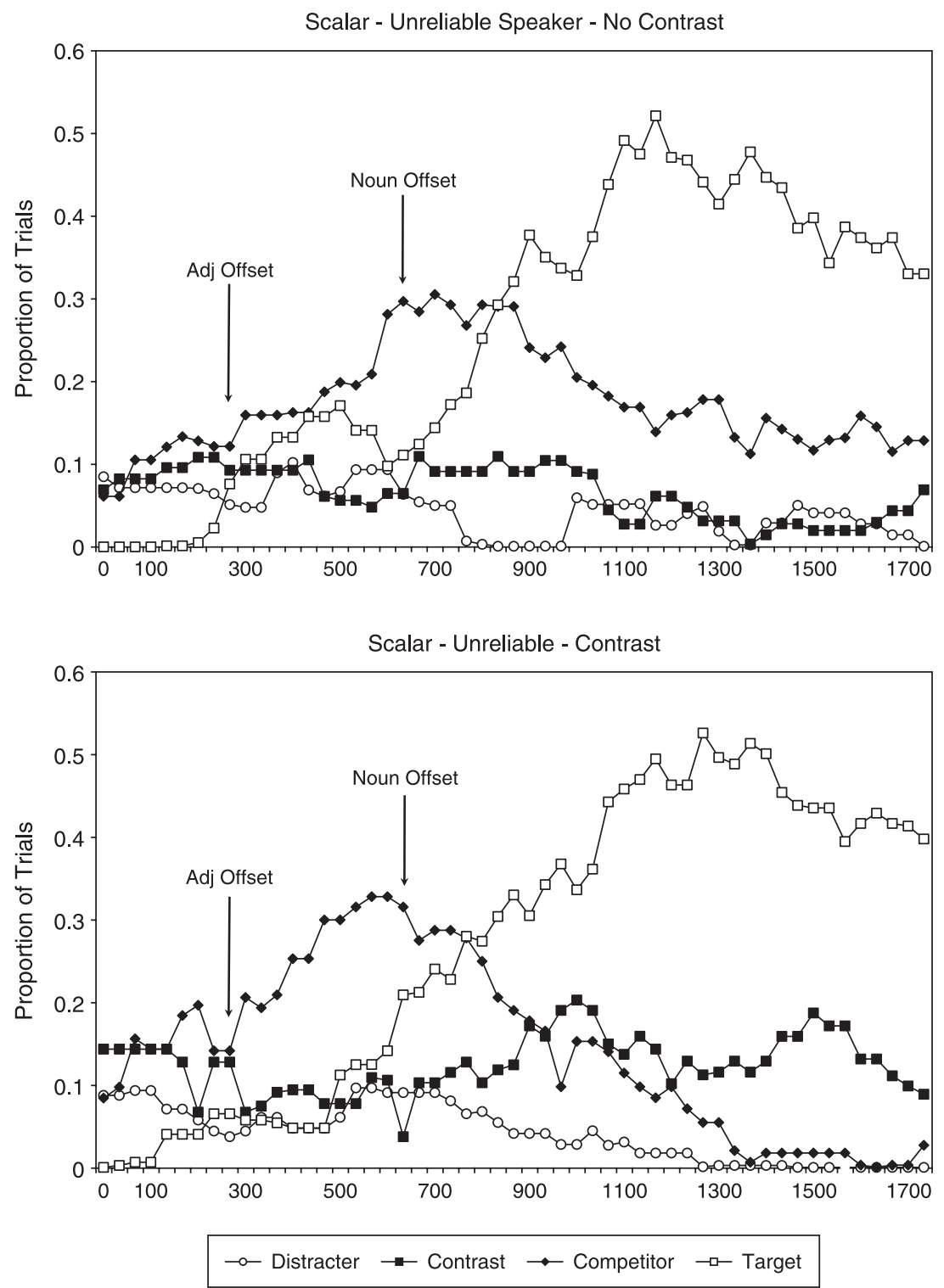

Figure 10.2

(continued) 
allowed for the alignment of eye movements to speech, as presented in the Results section below. For purposes of analysis, the work surface was divided into a $3 \times 3$ grid with visibly demarcated boundaries. Eye movements to an object were coded from the first frame in which a saccade was launched to the square containing that object. Occasionally, poor calibration or eye blinks resulted in a temporary loss of tracking. If tracking resumed less than five video frames later and reappeared on the same location, the track was treated as continuous; otherwise, the eye-movement record noted the loss of tracking and, for that time period, treated the fixation as falling on none of the objects in the display.

\section{Results}

Trials in which a participant reached for the incorrect object or the participant fixated on the target at the beginning of the adjective were omitted from analyses. The latter restriction was intended to exclude fixations on the target that were not initiated on the basis of speech input. Further, data points more than two standard deviations away from the mean for each condition and time frame were replaced with the mean for that condition. This affected 3.2 percent of the data.

Figure 10.2 depicts the proportion of trials including fixations to each of the objects in the display from the onset of the adjective. To correct for variability in the auditory duration of the modifier across stimuli, each trial was aligned to the offset of the adjective. Average offsets for the noun and the adjective in each condition are indicated. Critical comparisons were conducted over the 500 -msec window beginning $200 \mathrm{msec}$ after adjective offset. This corresponds to where manipulations of discourse contrast have been observed in previous work. Analyses were performed for each modifier type separately. For scalar items, target advantage scores were computed by subtracting fixations to the competitor from fixations to the target over the critical interval. This provided a composite measure of the relative proportion of fixations to the entities that should be affected by the presence of a referential contrast. Figure 10.3 depicts target advantage scores for the scalar items. A $2 \times 2$ ANOVA crossing speaker type and the presence or absence of a contrasting object in the display resulted in an interaction significant in the participants analysis $(\mathrm{F} 1(1,29)=5.05$, $\mathrm{MSE}=.043, p<.05)$, but not in the items analysis $(\mathrm{F} 2(1,9)=2.24, \mathrm{MSE}=.032$, $p=.17)$. Planned comparisons for each type of speaker revealed that perceivers responding to the reliable speaker benefited from the presence of a contextual contrast $(\mathrm{F} 1(1,14)=17.98, \mathrm{MSE}=.024, p<.001 ; \mathrm{F} 2(1,9)=4.6, \mathrm{MSE}=.041$, $p<.05)$ but those responding to the unreliable speaker did not $(F$ 's $<1)$. To establish the relative contributions to this pattern of looks to the target and the 


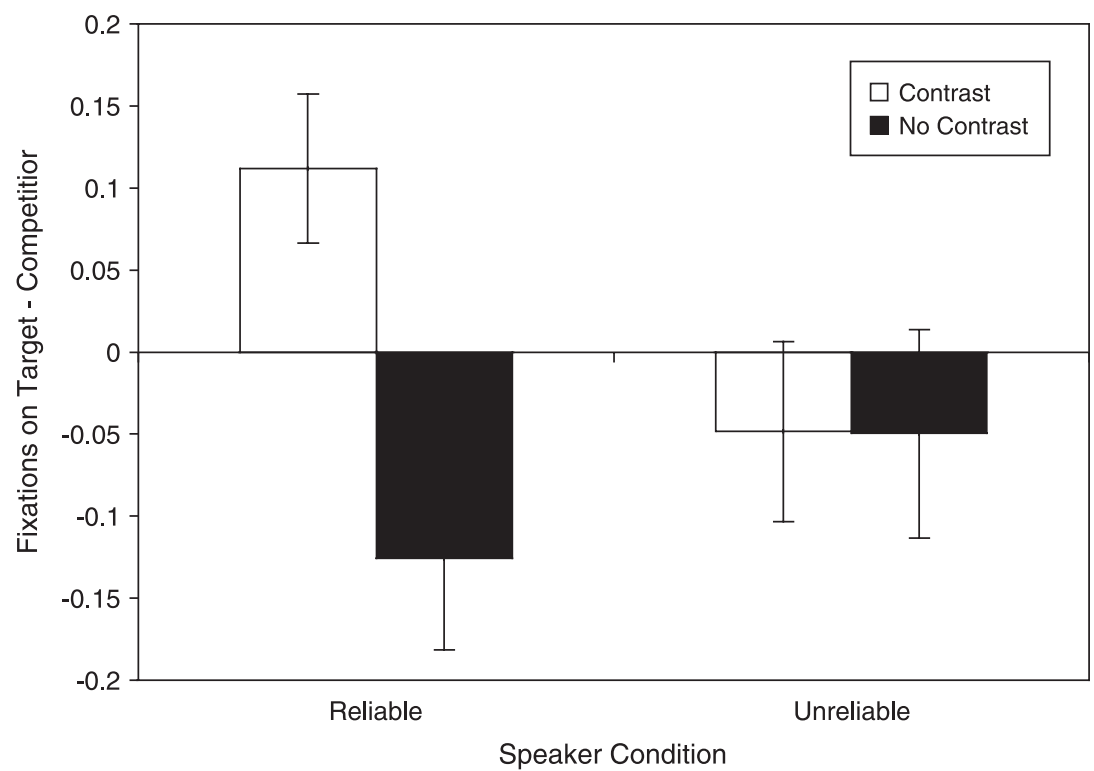

Figure 10.3

Target advantage scores for trials containing scalar adjectives over 500-msec interval region beginning $200 \mathrm{msec}$ after adjective offset.

competitor, additional analyses were conducted for fixations to each of these objects separately. Analysis of the proportion of fixations to the target patterned similarly to target advantage scores. There was a trend toward an interaction of speaker and contrast marginal by participants $(\mathrm{F}(1,29)=3.36, \mathrm{MSE}=$ $.01, p=.08)$, though not by items $(\mathrm{F} 2(1,9)=1.41, \mathrm{MSE}=.01, p=.26)$. Independent comparisons established that reliable speakers elicited significantly more looks to the target in the presence of a contrast $(\mathrm{F} 1(1,14)=11.9, \mathrm{MSE}=$ $.007, p<.01 ; \mathrm{F} 2(1,9)=6.4$, MSE $=.01, p<.05)$ and unreliable speakers did not $(F$ 's $<1)$. Proportions of fixations to the competitor also appear to have contributed to the interaction of target advantage scores. There was a marginal interaction by participants $(\mathrm{F} 1(1,29)=4.07, \mathrm{MSE}=.019, p=.05)$, but not by items $(\mathrm{F} 2(1,9)=1.44, \mathrm{MSE}=.021, p=.26)$. For the reliable speaker, there were fewer spurious looks to the competitor in the presence of a contrast $(\mathrm{F} 1(1,14)=15.2, \mathrm{MSE}=.008, p<.01 ; \mathrm{F} 2(1,9)=2.2, \mathrm{MSE}=.026, p=.09)$. The manipulation of contrast did not affect fixations to the competitor for the unreliable speaker $(F$ 's $<.03)$.

Figure 10.4 portrays looks to objects in the display in response to instructions containing material modifiers. Target advantage scores were computed 

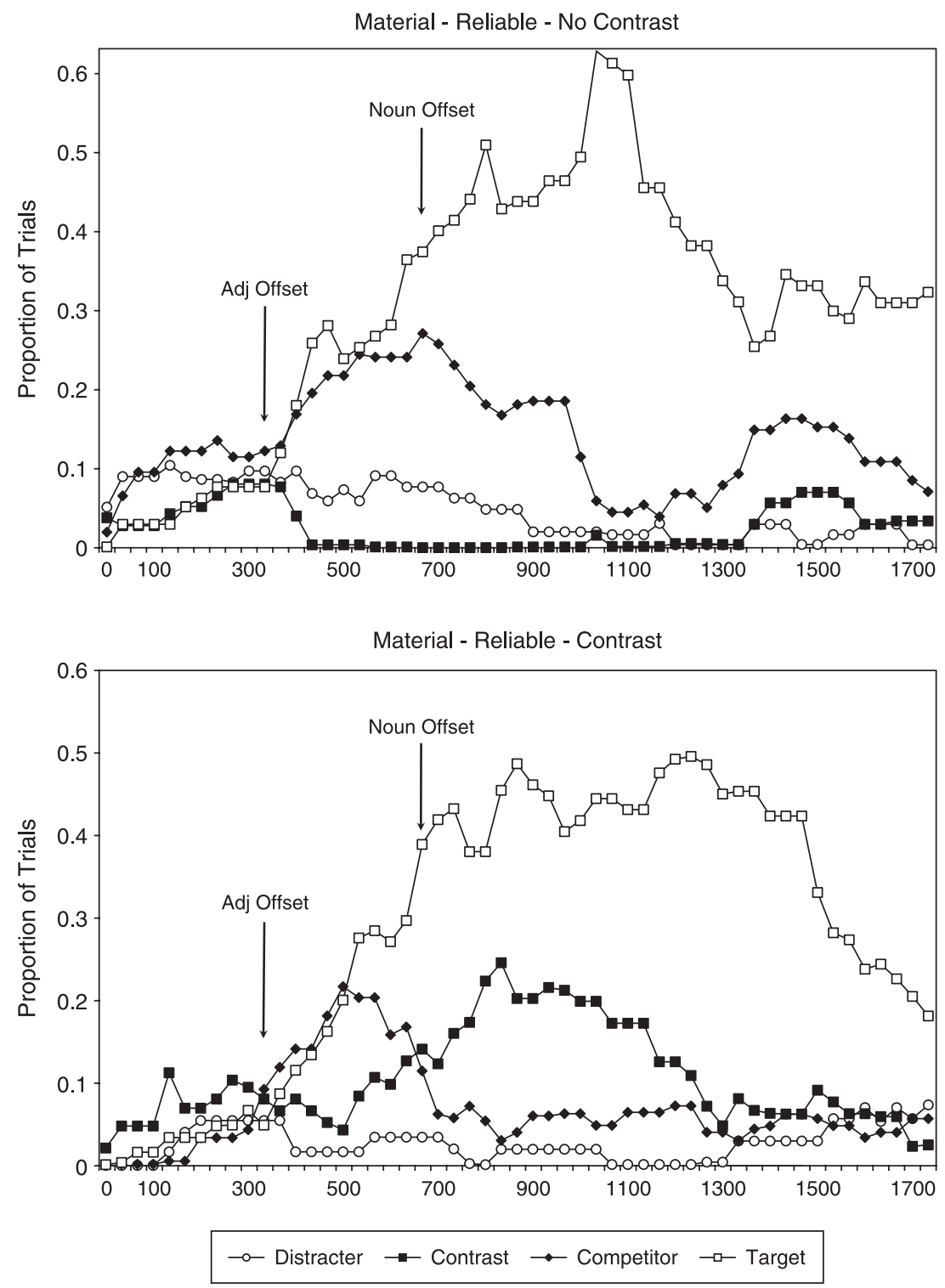

Figure 10.4

Eye-movement record for the items containing material adjectives. Horizontal axis depicts msec after adjective onset. In the no-contrast conditions the data labeled "contrast" indicate the second distracter item in the display. 

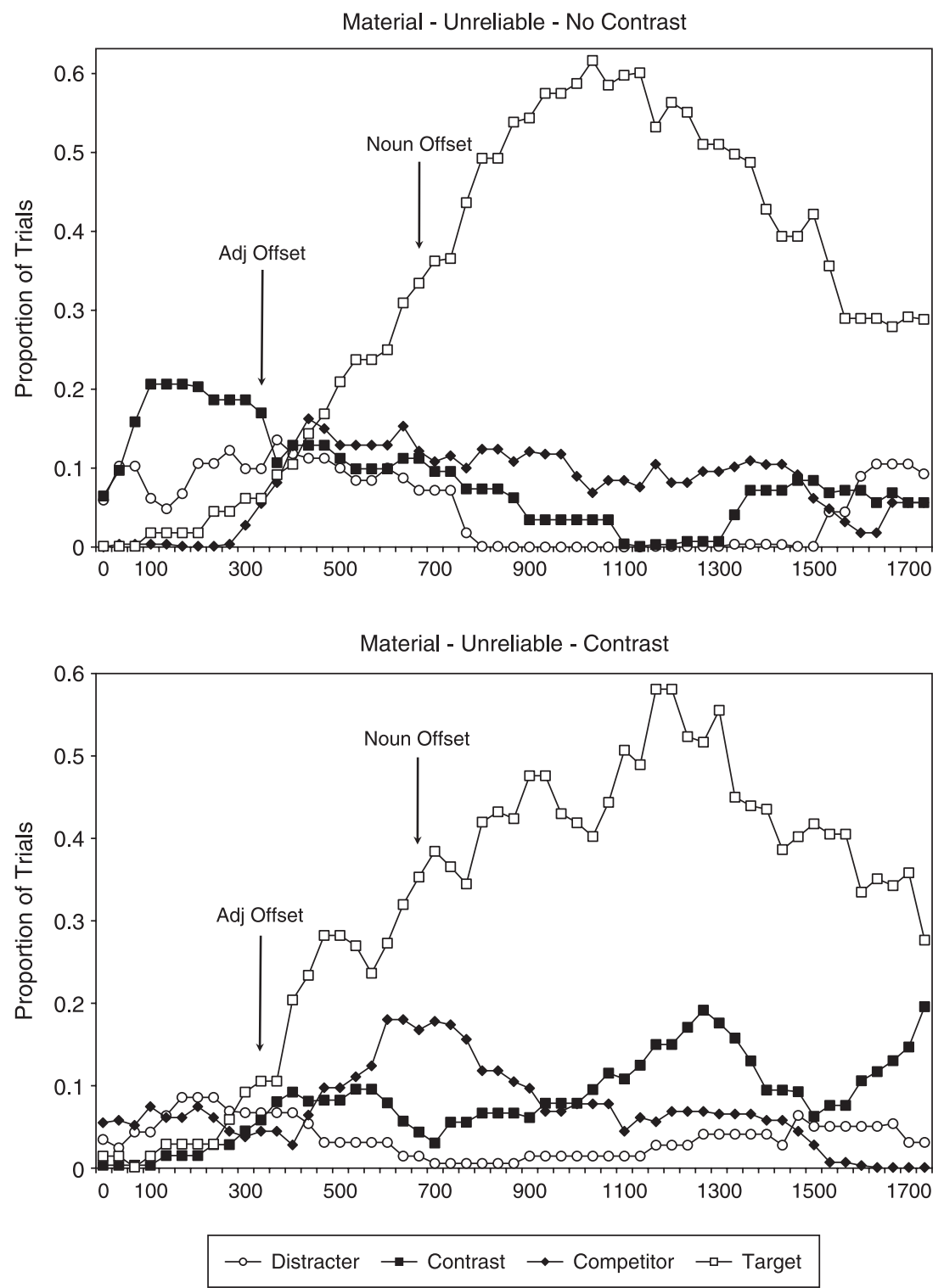

Figure 10.4

(continued) 


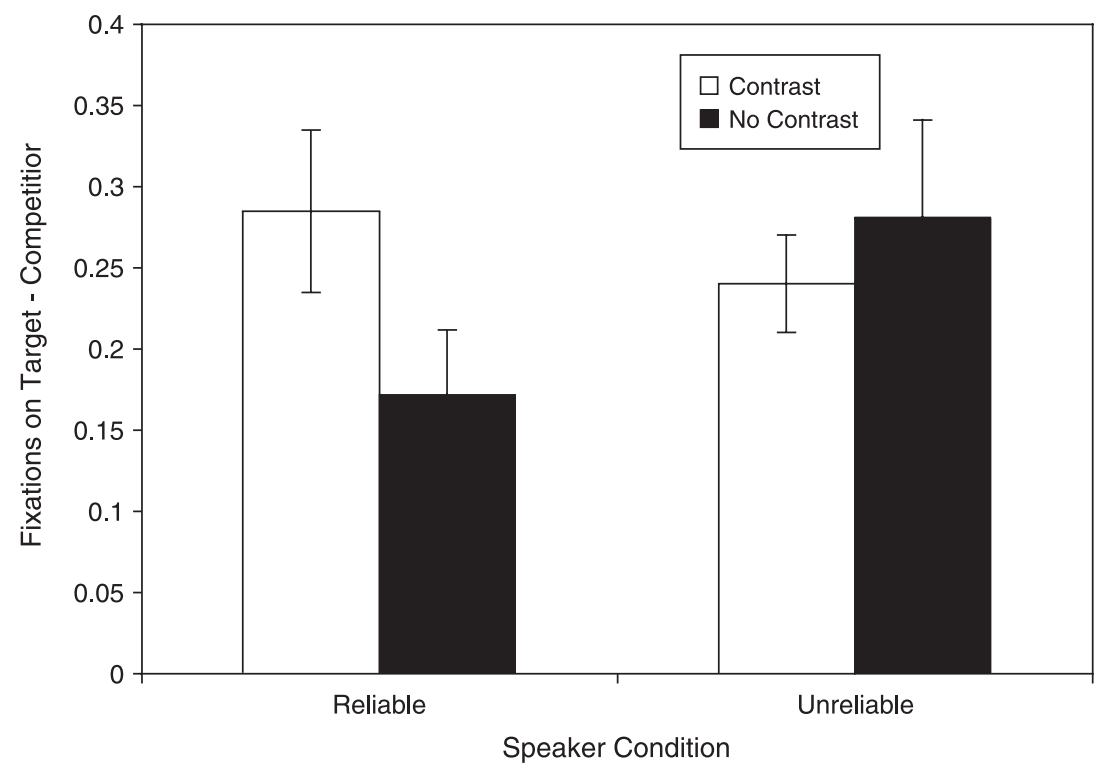

Figure 10.5

Target advantage scores for trials containing material adjectives over 500-msec interval region beginning $200 \mathrm{msec}$ after adjective offset.

and submitted to a $2 \times 2$ ANOVA crossing speaker reliability and contextual contrast (see figure 10.5). This revealed a significant interaction by items $(\mathrm{F} 2(1,9)=9.03, \mathrm{MSE}=.011, p<.05)$, but not by participants $(\mathrm{F} 1(1,29)=1.92$, $\mathrm{MSE}=.048, p=.18)$. Planned comparisons demonstrated a marginally reliable trend for higher target advantage scores in the presence of a contrast for reliable speakers $(\mathrm{F} 1(1,14)=2.63, \mathrm{MSE}=.036, p=.06 ; \mathrm{F} 2(1,9)=2.3, \mathrm{MSE}=$ $.024, p=.08)$. In contrast, target advantage scores were numerically lower in the unreliable-speaker condition, though this trend was not reliable $\left(F^{\prime}\right.$ 's $\left.<2.8\right)$. Just as for the scalar conditions, looks to the target and the competitor were analyzed separately. There were no clear effects or interactions for fixations to the target $(F$ 's $<.5)$. The absence of these effects and the relatively high target advantage scores likely reflect the tendency for individuals to identify the target extremely rapidly for the material modifiers. Looks to the competitor therefore provide a more sensitive indicator of the effect of contrast. Speaker type and contrast interacted reliably $(\mathrm{F} 1(1,29)=5.6, \mathrm{MSE}=.011, p<.05$; $\mathrm{F} 2(1,9)=18.4, \mathrm{MSE}=.003, p<.01)$. In response to reliable speakers, there were fewer looks to the competitor when a contrasting object was in the display $(\mathrm{F}(1,14)=7.64, \mathrm{MSE}=.013,=p<.05 ; \mathrm{F} 2(1,9)=18.1, \mathrm{MSE}=.004, p<.01)$. 
This was not the case for individuals in the unreliable-speaker condition $(F$ 's $<.75){ }^{3}$

It was possible that perceivers in the unreliable-speaker condition might have delayed interpretive processes in light of the irregular instructions. To ensure that this was not the case, analyses were performed to establish when combined fixations to the competitor and the target, each of which matched the modifier property, diverged from looks to other objects in the display. In response to the reliable speaker, participant fixations isolated the target and the competitor with marginal reliability between 67 and $100 \mathrm{msec}$ after adjective offset $(\mathrm{F}(1,14)=2.36, \mathrm{MSE}=.009 p=.07)$. For instructions containing scalar modifiers, this divergence first occurred in the window between 200 and 233 msec after adjective onset $(\mathrm{F}(1,14)=2.43, \mathrm{MSE}=.009, p=.07)$. For material modifiers, the difference was first observed between 33 and $66 \mathrm{msec}(\mathrm{F}(1,14)=$ 2.04, $\mathrm{MSE}=.011, p=.09$ ). Adjective-linked eye movements in response to the unreliable speaker were initially observed between 67 and $100 \mathrm{msec}$ over all stimulus items $(\mathrm{F}(1,15)=2.87, \mathrm{MSE}=.005, p=.06)$, between 100 and 133 msec for scalar conditions $(\mathrm{F}(1,15)=2.04, \mathrm{MSE}=.012, p=.09)$, and between 67 and $100 \mathrm{msec}$ for the material conditions $(\mathrm{F}(1,15)=3.53$, MSE $=.033$, $p<.05)$. For every comparison given here, fixations to the target and the competitor were significantly higher than to other objects in the display at each subsequent 33-msec analysis frame $(F$ 's $>3.5, p$ 's $<.05)$. In view of estimates that programming and launching a saccade takes approximately $200 \mathrm{msec}$ (Matin, Shao, and Boff 1993), these comparisons indicate that participants in both conditions are using the speech input to incrementally fix reference before the offset of the modifier. Importantly, participants in the unreliable-speaker condition were at least as rapid to respond to the literal denotation of the modifier (and, in the case of the scalar adjectives, perhaps quicker) as those in the reliable-speaker condition. The unnaturalness of the speaker did not cause interpretation to be any less incremental. Note too that eye movements were not retarded by the slightly faster material adjectives in the unreliable-speaker condition.

\section{Discussion}

The results demonstrate that speaker-specific attributes influence whether a restrictive modifier will be interpreted contrastively. In line with previous work, individuals in the reliable-speaker condition were aided in determining the referent for a restrictively modified nominal description by the presence of a contextual contrast. With scalar modification, there were more early looks to the target, and fewer looks to a competing object that matched the scalar property. For the unreliable speaker, neither effect was observed. When the reliable 
speaker uttered descriptions containing material modifiers, fewer looks were elicited to the competing object in the presence of a contrasting object. This too was not observed with the unreliable speaker. Thus, individuals were not aided by a contextual contrast when they had reason to believe a speaker did not use modification cooperatively. This is in accord with the view that participants in the unreliable-speaker condition did not take the presence of a modifier to imply the existence of a contrast set. These participants were just as responsive to the literal meaning of the adjective as for the reliable speaker. The speaker manipulation selectively eliminated the generation of the contrastive implicature licensed by the adjective.

That contrastive inferences can be eliminated by manipulating speaker reliability provides strong support for a pragmatic interpretation of referential contrast effects, and furthermore implies that early inferencing admits episodic information. The speed of contrastive inferences therefore cannot be explained as an automatic reflex of deviating from an immutable default form. This is at odds with the most straightforward reading of the short-circuited implicature proposal introduced above. However, this result does not imply that individuals are engaging in an overt counterfactual deductive reasoning process in order to use the contextual contrast appropriately. We return to this point in the general discussion.

Each modifier condition was similarly affected by speaker reliability and by contextual contrast. The presence of a contrast improved target identification for both scalar and material conditions in response to reliable speakers. In both cases, the effect of referential contrast vanished with unreliable speakers. Thus the contrastive inference is pragmatic in origin for relational (scalar) and non-relational (material) modifiers alike. This rules out the possibility that scalar terms convey the expectation for contextual contrast as part of their literal meaning. If they did, then the contrast effect should not have been canceled by manipulating the pragmatic context of the modifier's use. This is not to say that scalar and material adjectives were interpreted identically. Individuals were somewhat slower to map scalar meanings to the subset of items that matched the modifier denotation. It is possible that the relational component of scalar meaning complicates the task of converging on a target. Parallel findings from a recent production study conducted in our laboratory found that more disfluencies occur before prenominal scalar modifiers than before material or color modifiers (Gregory, Grodner, Joshi, and Sedivy 2003). This is particularly striking in light of the fact that scalar terms are more frequent in both spoken and written corpora. Taken together, these observations buttress the thesis that scalar denotations are more conceptually complex than non- 
comparative adjective denotations. Investigating how the lexical semantics of various modifiers mediates reference resolution is an interesting direction for future research.

\section{General Discussion}

The results reported herein strengthen the case for a pragmatic explanation of referential contrast effects by demonstrating their defeasibility. In addition, they indicate that there is a limit to the generality of quantity-based inferences and that characteristics particular to a speaker are taken into account in the generation of referential contrast inferences. A number of questions remain open. For example, the results reviewed above do not address how perceivers' expectations are updated or represented in a way that can influence early inferencing. It is interesting to speculate which cue to unreliability, or combination of cues, caused the attenuation of the contrast effect. One possibility is that perceivers' inferences hinge directly on their overt beliefs about the degree to which the speaker is conforming to principles of rational, orderly communication. Hence, an awareness of the mistakes made by the speaker and the explicit identification of the speaker as non-normal defuse the contrastive inference. Perceivers may believe that the speaker's impairment causes him to be an uncooperative or unreliable communicative partner and thus suspend any inferences made on that basis.

A second possibility is that pragmatic inferencing reflects a more implicit assessment of the communicative proclivities of the speaker. For instance, perceivers may attend to the statistics of modifier use for a particular speaker. Over the course of the experiment they would note that the presence of the adjective is not a reliable cue to contrast. As a result of the overuse of the modifier, they might recalibrate the anticipated default description to include a modifier. This view predicts that the effect of contrast might get weaker with repeated exposure to over-descriptive referring expressions (though it is unclear how much local experience might be necessary to override the prepotent contingency between modification and contextual modification). Anecdotal evidence from post-experimental debriefing suggests that perceivers do not register conscious awareness of the extent to which speakers exhibit optimally informative communicative behavior. When queried whether they noticed anything unusual about the experiment, participants frequently mentioned trials in which objects were mislabeled or in which object destinations made reference to impossible configurations. They rarely if ever mentioned the over-explicit object labels. These observations hint that the speaker's overspecified descriptions did not have a large impact on the overt assessment of the reliability of 
the speaker. Still, the speaker-specific modulations of the contrast effect could be attributable to high-level beliefs about speaker characteristics, lower-level statistical properties of the speakers output, or some combination of both. If only high-level cues are needed to indicate speaker unreliability, then perceivers need not learn about the particular way in which the present speaker is uninformative, and should suspend the computation of a broad range of implicit meanings. Further, there should be no evidence of greater reduction in the contrast effect as the perceiver accumulates evidence of over-informativity. On the other hand, sensitivity to lower-level cues should result in incremental changes to how perceivers respond to the descriptive patterns used by a speaker as more tokens of speaker descriptions are encountered.

To evaluate these alternatives, exploratory analyses were conducted comparing performance on items in the first and the second halves of the experiment. The hypotheses differ with respect to their predictions for the reliable-speaker conditions. To see why this is so, note that reliable speakers uttered modified forms to refer to competitors as often as they did to pick out a member of a contrasting pair (for ten items across the 56 experimental trials in each case). Hence, there was no reliable contingency between modification and contextual contrast for the reliable-speaker condition. Thus, over the course of the experiment, perceivers may have come to adjust their expectations about the information conveyed by modification as a result of encountering a significant number of modified forms in the absence of contrast. The unreliable speaker, on the other hand, consistently used over-specific forms on a much large number of trials, with 15 modified forms occurring in the absence of contrast even before the first experimental item. Therefore, statistics corresponding to the overuse of the modifier may have already been adjusted on the expected referential form by the point at which inferential processes could be assessed in the first half of the experiment.

The most interesting measure for the reliable speaker was looks to the competitor for scalar items, depicted in figure 10.6. The interaction of contrast and block order was marginal $(\mathrm{F}(1,13)=3.61, \mathrm{MSE}=.021, p=.08)$. Consistent with the statistical tuning hypothesis, looks to the competitor were reduced in the presence of a contrast in the first block of the experiment $(\mathrm{F}(1,13)=7.41$, MSE $=.031, p<.05)$, but not in the second $(\mathrm{F}<1)$. There were no effects of contrast or interactions with block order for the unreliable speaker $(F$ 's $<1.5)$. Two caveats are in order: The present experiment was not specifically designed to test these hypotheses and no other measure differed reliably across blocks. Still this trend is suggestive that overuse of the modifier contributes to the elimination of the contrast effect. 

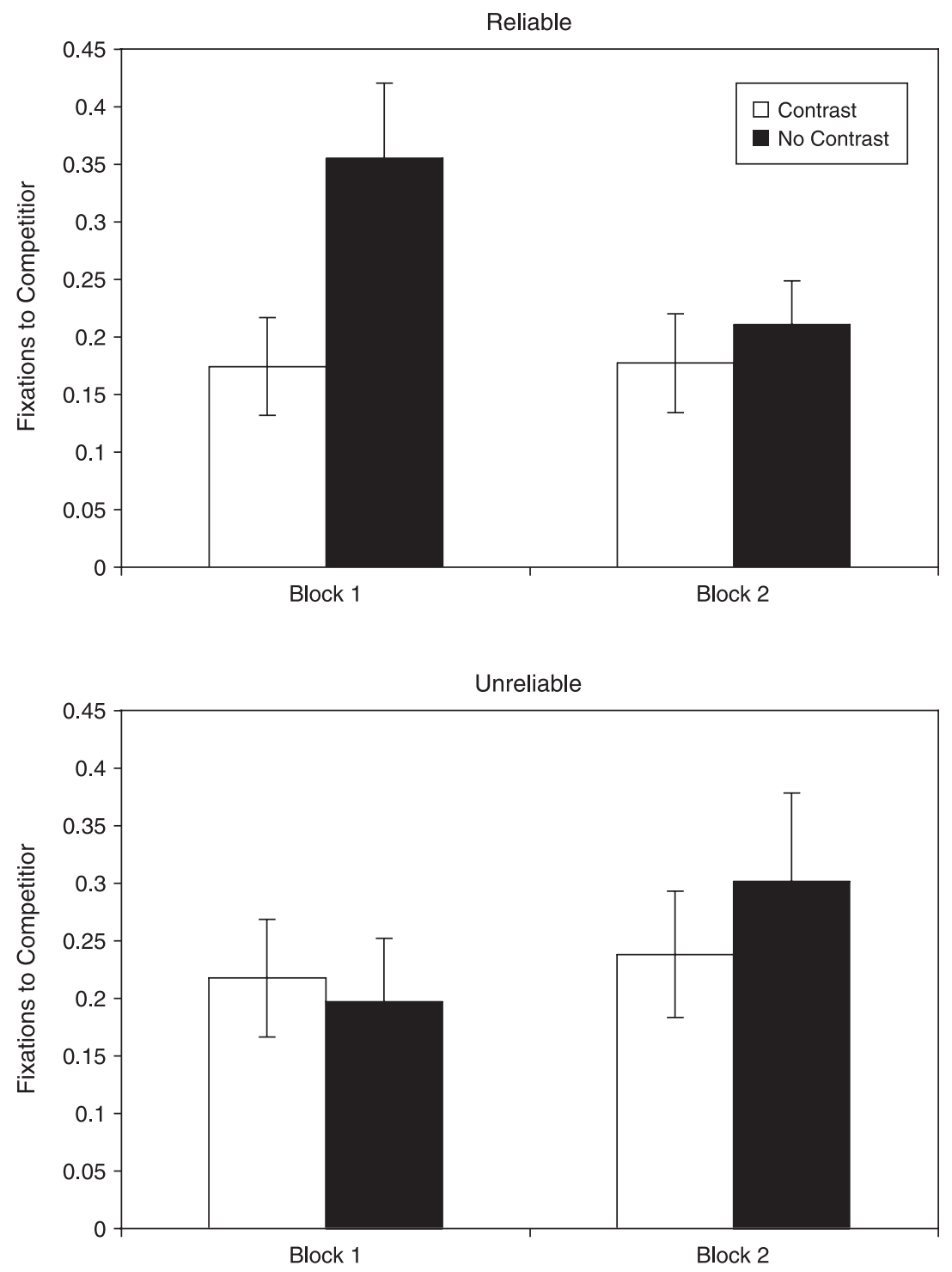

Figure 10.6

Looks to competitor object over interval 200-700 msec after noun onset for scalar items across first and second blocks of experiment. Top and bottom panels represent data from the reliable and unreliable speaker conditions, respectively. 
A follow-up study delved further into the source of the pragmatic effect. A reliable-speaker condition was compared against two unreliable speakerconditions. In one of these unreliable conditions, the speaker overused prenominal modifiers consistently just as in the present experiment. In the other, the speaker encoded the same redundant content as the prenominal condition, but did so with a postnominal modifier (e.g., "Pick up the cup that's tall"). As in the previous experiment, critical trials tested instructions containing prenominal modifiers for all three conditions. But in contrast with the first experiment, none of the explicit cues to speaker irregularity were given. If perceivers are sensitive to the redundancy of content, then both unreliable speaker types should result in a reduction of the contrast effect. If perceivers are especially sensitive to a redundancy of a particular form, then the excessive use of the prenominal modifier should result in a greater reduction of the contrast effect than the postnominal condition. It is also possible that high-level knowledge of the speaker's impairment would be necessary to draw attention to low-level redundancies in the present study. If so, then there might not be a marked reduction in the effect of contrast.

Figure 10.7 depicts target advantage scores for instructions containing scalar terms over the same temporal region analyzed in the present experiment. For all three conditions there was an apparent benefit of the presence of a contrast in identifying the target. This was significant by participants and items for the reliable speaker $(\mathrm{F} 1(1,18)=5.16, \mathrm{MSE}=.05, p<.05 ; \mathrm{F} 2(1,9)=5.48, \mathrm{MSE}=$ $.02, p<.05)$, and by participants for the condition with excessive prenominal modification $(\mathrm{F} 1(1,18)=3.87, \mathrm{MSE}=.03, p<.05 ; \mathrm{F} 2(1,9)=1.28, \mathrm{MSE}=.03$, $p=.14)$. Though a similar trend was observable for the condition with excessive postnominal modification, it was marginally reliable only in the items analysis $(\mathrm{F} 1(1,16)=1.59, \mathrm{MSE}=.09, p=.11 ; \mathrm{F} 2(1,9)=2.44, \mathrm{MSE}=.04, p=$ $.08)$. There was no hint of an interaction between speaker type and contrast $(F$ 's $<.5)$. This work demonstrates that overusing restrictive modification is not sufficient by itself to eradicate the contrastive interpretation of scalar modifiers. This does not immediately imply that the high-level cues to speaker reliability were solely responsible for defusing the expectation of contrast in the earlier experiment. It is possible that high-level cues were necessary to draw the listener's attention to the low-level redundancy. It is also possible that the pattern observed in the follow-up experiment was unique to scalar modifiers, which are inherently comparative.

Another open question is what kind of pragmatic mechanism could be responsible for the above findings. A Gricean explanation for contrastive interpretations of restrictive modifiers claims that perceivers make the following inferential steps when a speaker utters a modified description: 


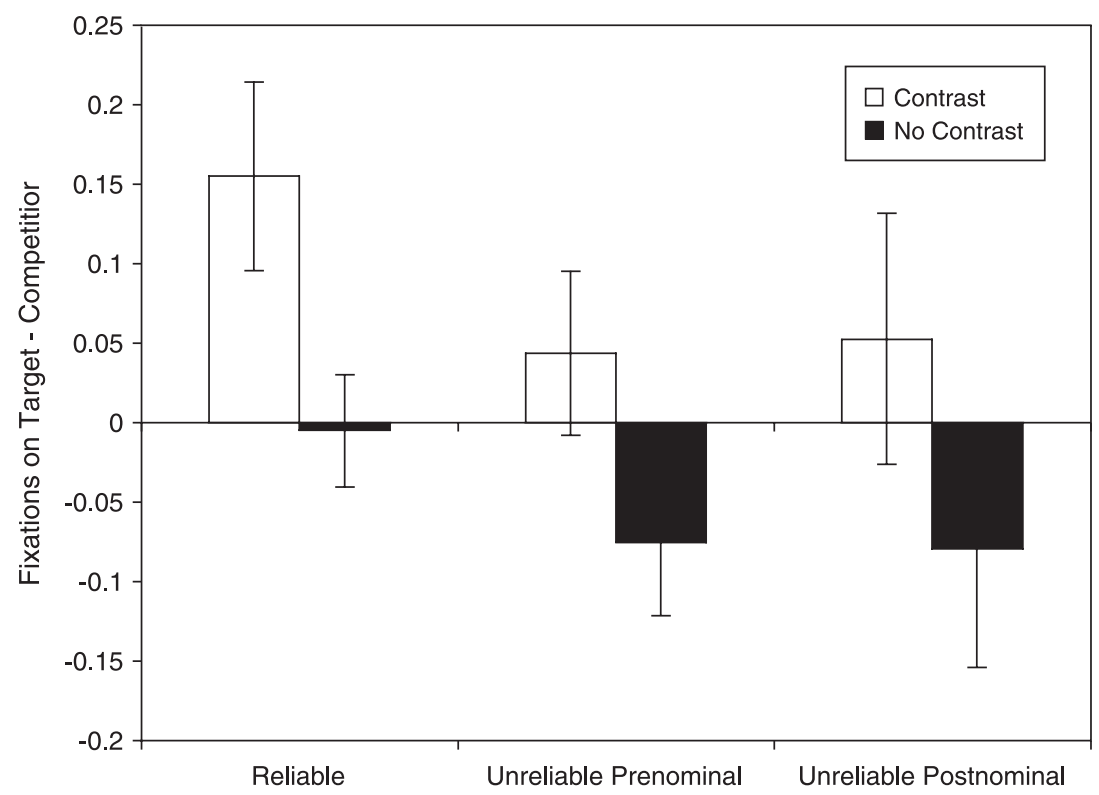

Figure 10.7

Target advantage scores elicited by instructions containing scalar terms uttered by a speaker who use modification reliably $(N=19)$, a speaker who used prenominal modification excessively $(N=19)$, and a speaker who used postnominal modification excessively $(N=17)$.

(i) If the speaker means to use this utterance to pick out an intended referent in a context with only one entity of that type, then a default description would be the most natural means to do so.

(ii) The speaker chose a more specific description than the default by using a modified referential phrase.

(iii) If the speaker is behaving cooperatively, he should not be more informative than is necessary. Overspecification must have some purpose.

(iv) Because of (ii) and (iii), the conditions for uttering a default form in (i) must not hold. That is, it must not be the case that perceiver intends the utterance of the modified form to pick out an entity in isolation.

One possible conclusion of (i)-(iv) is (v):

(v) There are multiple entities in the context of the same type.

There are a number of challenges inherent to implementing this sort of reasoning in a real-time processing system. For one, it is not clear what kind of reasoning system would permit arriving at (v) as rapidly as perceivers do. That is, 
given step (iii), what ranges of "purposes" for the inclusion of modification are considered, and what weight is each of them given? It is certainly not the case that distinguishing between like entities is the only or even the most frequent function of modification. (See Fox and Thompson 1990 for a taxonomy of functions of relative-clause modifiers.) Second, a critical step is embedded in (ii). How does the system decide the appropriate alternative to compute and compare to a given referential expression? For instance, "the plastic cup" is a more specific description than "the plastic entity," yet intuition tells us that the contrastive entity invoked is another cup and not another plastic object. The comparison of alternative forms is even more impressive when we consider that it is occurring incrementally even before the noun is encountered. A partial answer to both of these questions might be that statistical regularities constrain the consideration of alternative expressions and their functions, and indeed that inferential steps may be statistically linked. This would permit the processor to bypass the complexity inherent in reasoning about alternatives counterfactually.

Let us sketch one way this approach might work to account for the currently known facts about contrastive inferences. First, determining the referential forms to be compared could be a function of patterns of co-occurrence among properties in referential descriptions. Modifiers are more promiscuous than nouns. "Plastic" will be used to modify a wide variety of artifact labels, whereas "cup" will be used to describe a narrow set of object referents. The most accessible default for "the plastic cup" is "the cup" rather than "the plastic thing" because the descriptions in which "cup" participates form a more coherent set of properties than the descriptions in which "plastic" participates. This would explain our intuitions about the dimension along which a contrast set differs from the referent set. Whether or not an expression that is actually used deviates from the expected default could be computed from the likelihood of using a modifier encoding some specific property together with a particular noun. For instance, the ratio of expressions encoding yellowness as a property given the total occurrences of a noun such as "banana" would presumably be lower than the ratio of encoding "yellow" given "notebook." Thus, "yellow banana" would represent more of a deviation from the expected default than "yellow notebook," triggering a search for some function to the modifier. The preferred function (i.e. referentially contrasting) could be arrived at via a statistical link between steps (ii) and (v) (though note that this statistical link remains to be empirically established, given the observations in Fox and Thompson). Clearly this is an extremely partial sketch of how the aforementioned challenges might be met. Our present purpose is merely to point to potential directions for realizing a reasoning system that is flexible enough to 
consider situation-based information, but sufficiently constrained to operate in real time.

There are at least two points in the process outlined above at which speakerspecific effects could have influenced the present experiment. One is to directly recalibrate the default form at step (ii) for a particular speaker on the basis of statistical regularities in the recent episodic record. An alternative that seems more computationally cumbersome is to generate expectations of defaults more generally in step (ii) as a function of global experience across many speakers, and then to invoke the criterion in (iii) as a prerequisite to identifying the most likely function for the modifier. In the former case, speaker-particular regularities for the speakers in the experiment reported above might be used to calibrate expectations about default expressions. That is, modified descriptions would count as deviations from the expected default for reliable speakers, but not for unreliable speakers. This type of rapid calibration to characteristics of a speaker's speech could be similar to the low-level acoustic calibration that is automatically achieved to take into account speaker characteristics such as age and gender, allowing perceivers to cope efficiently with speaker variability. The second alternative posits that the use of a modified phrase would count as a deviation from the default in both speaker conditions; however, step (iii) would then be invoked, and the determination of a speaker's unreliability with respect to communicative norms would suspend a search for an appropriate function for the modifier. This latter explanation is more computationally complex, and is not able to exploit direct statistical links between steps (ii) and (v).

Our follow-up experiment attempts to distinguish between these two explanations. Thus far, the results suggest that a determination of speaker reliability (i.e. step (iii)) may not be entirely dispensable in accounting for contrast set inferences. Further research is needed to determine whether overt signaling of speaker unreliability or uncooperativeness is both necessary and sufficient to suspend the process of forming contrastive inferences, as well as what sorts of evidence can be used to make this determination.

Another way of exploring the explanatory power of statistical regularities in generating pragmatic inferences is to examine whether perceivers' generation of a contrastive implicature depends on the identification of plausible alternative functions of modifiers. To test this theory, it would be interesting to see if a pragmatic manipulation that did not alter the cue validity of a modifier could defuse the contrastive implicature. Consider the sentence in (5) uttered within a discourse where a woman is trying and failing to reach a cup on a shelf.

(5) The short woman could not reach the cup on the top shelf. 
The modifier provides a causal explanation for the prominent event. Intuitively no contrasting woman is conjured by this example. It is possible that the prepotent identifying function of the modifier initially results in a contrastive interpretation, which is later retracted on the basis of secondary deliberative processes. Alternatively, one might see no evidence of contrast inferences in initial referential commitments, which would suggest that the identification of alternative functions comes into play before the conclusion reached in step (v). This could be implemented either at step (ii), in allowing relevance-based considerations to constrain expected default expressions, or at step (iii), once a deviation from the expected expressions triggers a search for plausible functions for that deviation. Careful temporally sensitive experimentation along these lines has the potential to clarify the computation of pragmatic inferences that have hitherto been addressed primarily by theoretical linguists. Representational distinctions have been posited between encoded content implicit content, and between generalized and particularized implicature. These formal distinctions provide useful starting points for formulating processing hypotheses. Further investigation along these lines will allow us to gain ground in understanding which parts of the inferential process are generated automatically and which parts are computed ad hoc.

\section{Acknowledgments}

This work profited from helpful comments and feedback given by the audiences at the 16th CUNY conference on Sentence Processing in Cambridge and the 8th AMLaP Conference in Glasgow. We are grateful to Anjula Joshi, Natasha Trentacosta, and Michele Hebert for assistance in collecting and coding data. This work was supported in part by NIH grant F32 MH65837-01 awarded to the first author and NIH grant R01 MH62566-01 awarded to the second author.

\section{Notes}

1. Here and throughout, we use the term "inference" to refer to information that is communicated to the perceiver via the utterance of an expression, but which is not part of its asserted content. This would include both accommodated presuppositions and implicatures. We adopt this term because it is neutral with respect to whether the inferred content arises from the conventional or implicated meaning of the critical expression.

2. This example is adapted from Levinson 2000.

3. Figure 10.4 suggests that there were frequent looks to the second distracter (labeled as "contrast") before adjective offset in the unreliable-speaker no-contrast condition. Though proportion of fixations appears to be elevated relative to looks to the other 
distracter, this difference is not significant $(F$ 's $<1.7)$. There are also no such elevated fixations for the reliable-speaker no-contrast condition. Further, the elevation occurs extremely early ( $200 \mathrm{msec}$ before adjective offset), which makes it unlikely that it was affected by the critical description.

\section{References}

Altmann, G., Garnham, A., and Dennis, Y. 1992. Avoiding the garden-path: Eye movements in context. Journal of Memory and Language 31, 685-712.

Altmann, G., and Steedman, A. 1988. Interaction with context during human sentence processing. Cognition 30, 191-238.

Barr, D. J. 2003. Listeners are mentally contaminated. Poster presented at 44th annual meeting of Psychonomic Society, Vancouver.

Barr, D. J., and Keysar, B. 2002. Anchoring comprehension in linguistic precedents. Journal of Memory and Language 46, 391-418.

Bierwisch, M. 1987. The semantics of gradation. In M. Bierwisch and E. Lang (eds.), Dimensional Adjectives. Springer-Verlag.

Bradlow, A. R. 2002. Confluent talker- and listener-related forces in clear speech production. In C. Gussenhoven and N. Warner (eds.), Laboratory Phonology 7. Mouton de Gruyter.

Carston, R. 1998. Pragmatics and the Explicit-Implicit Distinction. Ph.D. thesis, University College London.

Chambers, C. G., Magnuson, J. S., and Tanenhaus, M. K. 2004. Actions and affordances in syntactic ambiguity resolution. Journal of Experimental Psychology: Learning, Memory and Cognition 30, 687-696.

Clifton, C., Jr., Bock, J., and Radó, J. 2000. Effects of the focus particle 'only' and intrinsic contrast on comprehension of reduced relative clauses. In A. Kennedy, R. Radach, D. Heller, and J. Pynte (eds.), Reading as a Perceptual Process. Elsevier.

Clifton, C., Jr., and Ferreira, F. 1989. Ambiguity in context. Language and Cognitive Processes 4 (special issue), 77-103.

Crain, S., and Steedman, M. 1985. On not being led up the garden path: The use of context by the psychological parser. In D. Dowty, L. Karttunnen, and A. Zwicky (eds.), Natural Language Parsing. Cambridge University Press.

Fox, B. A., and Thompson, S. A. 1990. A discourse explanation of the grammar of relative clauses in English conversation. Language 66, 297-316.

Gregory, M., Grodner, D., Joshi, A. and Sedivy, J. 2003. Adjectives and processing effort in production: So, uh, what are we doing during disfluencies? Paper presented at 16th annual CUNY conference on human sentence processing, Boston.

Grice, H. P. 1975. Logic and conversation. In P. Cole and J. Morgan (eds.), Syntax and Semantics 3: Speech Acts. Academic Press.

Hanna, J. E., Tanenhaus, M. K. and Trueswell, J. C. 2003. The effects of common ground and perspective on domains of referential interpretation. Journal of Memory and Language 49, 43-61. 
Isaacs, E. A., and Clark, H. H. 1987. References in conversations between experts and novices. Journal of Experimental Psychology: General 116, 26-37.

Jurafsky, D. 2003. Pragmatics and computational linguistics. In Laurence R. Horn and Gregory Ward (eds.), Handbook of Pragmatics. Blackwell.

Levinson, S. 2000. Presumptive Meanings. MIT Press.

Metzing, C., and Brennan, S. E. 2003. When conceptual pacts are broken: Partnerspecific effects in the comprehension of referring expressions. Journal of Memory and Language 49, 201-213.

Nadig, A. S., and Sedivy, J. C. 2002. Evidence of perspective taking constraints in children's on-line reference resolution. Psychological Science 13, 329-336.

Ni, W., and Crain, S. 1989. How to resolve structural ambiguities. In Proceedings of the 20th North East Linguistic Society Conference.

Ni, W., Crain, S., and Shankweiler, D. 1996a. Sidestepping garden paths: Assessing the contributions of syntax, semantics and plausibility in resolving ambiguities. Language and Cognitive Processes 11 (3), 283-334.

Rooth, M. 1985. Association with Focus. Ph.D. Dissertation, University of Massachusetts, Amherst.

Sadock, J. 1978. On testing for conversational implicature. In P. Cole (ed.), Syntax and Semantics, volume 9: Pragmatics. Academic Press.

Schober, M. F. 1998. Different kinds of conversational perspective-taking. In S. R. Fussell and R. J. Kreuz (eds.), Social and Cognitive Psychological Approaches to Interpersonal Communication. Erlbaum.

Sedivy, J. C. 2001. Evidence of Gricean expectations in on-line referential processing. Paper presented at 14th CUNY Conference on Sentence Processing.

Sedivy, J. C. 2002. Invoking discourse-based contrast sets and resolving syntactic ambiguities. Journal of Memory and Language 46 (2), 341-370.

Sedivy, J. C. 2003. Pragmatic versus form-based accounts of referential contrast: Evidence for effects of informativity expectations. Journal of Psycholinguistic Research $32(1), 3-23$.

Sedivy, J. C. 2005. Evaluating explanations for referential context effects: Evidence for Gricean mechanisms in on-line language interpretation. In J. Trueswell and M. Tanenhaus (eds.), World Situated Language Use: Psycholinguistic, Linguistic, and Computational Perspectives on Bridging the Product and Action Traditions. MIT Press.

Sedivy, J. C., Chambers, C., Tanenhaus, M., and Carlson, G. 1999. Achieving incremental semantic interpretation through contextual representation. Cognition 71, 109147.

Siegel, M. 1980. Capturing the Adjective. Garland.

Sperber, D., and Wilson, D. 1986. Relevance. Harvard University Press.

Spivey-Knowlton, M., and Tanenhaus, M. 1994. Referential context and syntactic ambiguity resolution. In C. Clifton, L. Frazier, and K. Rayner (eds.), Perspectives on Sentence Processing. Erlbaum. 
Steedman, M., and Altmann, G. 1989. Ambiguity in context: A reply. Language and Cognitive Processes 4 (special issue), 211-234.

Tanenhaus, M. 2003. Referential domains in language processing. Paper given at 2003 Architectures and Mechanisms of Language Processing conference, Glasgow.

Tanenhaus, M., Spivey-Knowlton, M., Eberhard, K., and Sedivy, J. 1995. Integration of visual and linguistic information in spoken language comprehension. Science 268, $1632-1634$. 